\title{
Vitamin D deficiency and the risk of tuberculosis: a meta-analysis
}

\author{
This article was published in the following Dove Press journal: \\ Drug Design, Development and Therapy \\ 28 December 2016 \\ Number of times this article has been viewed
}

\author{
Shao-Jun Huang' \\ Xian-Hua Wang ${ }^{2}$ \\ Zhi-Dong Liu' \\ Wen-Li Cao ${ }^{3}$ \\ Yi Han' \\ Ai-Guo $\mathrm{Ma}^{2}$ \\ Shao-Fa Xu' \\ 'Department of Thoracic Surgery, \\ Beijing Chest Hospital, Capital \\ Medical University, Beijing, People's \\ Republic of China; ${ }^{2}$ Department \\ of Nutrition and Food Hygiene, \\ Institute of Human Nutrition, Medical \\ College of Qingdao University, \\ Qingdao, People's Republic of China; \\ ${ }^{3}$ Department of Pulmonary Medicine, \\ Beijing Geriatric Hospital, Beijing, \\ People's Republic of China
}

Background and aim: To conduct meta-analyses of all published studies on various aspects of association between vitamin D and tuberculosis (TB).

Methods: PubMed and Web of Knowledge were searched for all properly controlled studies on vitamin D and TB. Pooled odds ratio, mean difference or standardized mean difference, and its corresponding 95\% confidence interval were calculated with the Cochrane Review Manager 5.3.

Results: A significantly lower vitamin D level was found in TB patients vs controls; vitamin D deficiency (VDD) was associated with an increased risk of TB, although such an association was lacking in the African population and in the human immunodeficiency virus-infected African population. A significantly lower vitamin D level was found in human immunodeficiency virus-TB-coinfected African patients receiving antiretroviral treatment who developed TB-associated immune reconstitution inflammatory syndrome vs those who did not develop TB-associated immune reconstitution inflammatory syndrome. VDD was associated with an increased risk of developing active TB in those subjects with latent TB infection and with an increased risk of tuberculin skin test conversion/ TB infection conversion, and the trend toward a lower vitamin D level in active TB patients vs latent TB infection subjects did not reach statistical significance, indicating that VDD was more likely a risk factor than a consequence of TB. This concept was further strengthened by our result that anti-TB treatment did not affect vitamin D level in TB patients receiving the treatment.

Conclusion: Our analyses revealed an association between vitamin D and TB. VDD is more likely a risk factor for TB than its consequence. More studies are needed to determine whether vitamin D supplementation is beneficial to TB prevention and treatment.

Keywords: vitamin D, vitamin D deficiency, tuberculosis, 25-hydroxyvitamin D, metaanalysis

\section{Introduction}

Tuberculosis (TB) remains a major public health problem globally. It was estimated that there were 8.7 million reported new TB cases and that 1.4 million people died from TB in 2011. ${ }^{1}$ Various factors that could possibly affect the incidence and progression of TB have been reported, one of them is vitamin D deficiency (VDD). ${ }^{1-6}$ Exposure to sunlight is the main source of vitamin D for human and induces the conversion of 7-dehydrocholesterol to vitamin D3 via previtamin D3 in the skin. ${ }^{7}$ Vitamin D3 is then converted to 25-hydroxyvitamin $\mathrm{D}(25(\mathrm{OH}) \mathrm{D})$ in the liver and is further converted to the bioactive form of vitamin D, 1,25-dihydroxyvitamin D3 (1,25(OH)2D3) in the kidney. ${ }^{7}$ Studies have found that $1,25(\mathrm{OH}) 2 \mathrm{D} 3$ binds to vitamin $\mathrm{D}$ receptor (VDR), activates VDR signaling, and induces a series of antimicrobial responses such as induction of autophagy, phagolysosomal fusion, release and activation of the antimicrobial peptide cathelicidin, and killing of intracellular Mycobacterium tuberculosis. ${ }^{7-9}$
Correspondence: Shao-Fa Xu Department of Thoracic Surgery, Beijing Chest Hospital, Capital Medical University, No 97 Machang,

Tongzhou District, Beijing,

People's Republic of China

Tel +86 I0 89509326

Email shaofaxu@yahoo.com
Drug Design, Development and Therapy 2017:1 | 91-102

91 
Numerous studies have been conducted to study whether VDD was associated with TB; however, they produced inconsistent and varying results. ${ }^{1-6,10-12}$ There has been one meta-analysis on association between VDD and TB, and it concluded that a lower serum vitamin D level was associated with a higher risk of active TB. ${ }^{13}$ However, it was conducted in 2008 and did not include numerous later studies, and further it did not perform a subgroup analysis based on factors such as ethnicity.

In this study, we performed meta-analyses comparing serum vitamin D levels in TB cases vs controls and on association between VDD and risk of TB with subgroup analysis based on the ethnicity of the study population in order to further elucidate the role of vitamin D level in TB pathogenesis in a larger population pool. Second, assuming VDD is indeed associated with TB, it is still undetermined whether VDD is one of the causes of TB or manifestation of the malnutrition status generally seen in TB patients. ${ }^{1}$ In order to explore this question, we further examined the association between vitamin D level/VDD and risk of developing active TB in subjects with latent TB infection (LTBI)/household contacts of TB patients and also the association between VDD and tuberculin skin test (TST) conversion/TB infection conversion (TBIC). An additional analysis was also performed on the effect of anti-TB treatment on vitamin D level in TB subjects for the same purpose. Third, since human immunodeficiency virus (HIV)-infected subjects are more susceptible to TB and have greater mortality and morbidity caused by $\mathrm{TB},{ }^{14}$ to further clarify whether vitamin D played any role in occurrence and deterioration of TB in HIV-infected subjects, we also analyzed the association between vitamin D level/ VDD and TB in HIV-infected subjects and the association between vitamin $\mathrm{D}$ level and risk of developing TB-associated immune reconstitution inflammatory syndrome (TB-IRIS) in HIV-TB-coinfected patients receiving antiretroviral therapy (ART). Finally, although serum $25(\mathrm{OH}) \mathrm{D}$ is the commonly used measure of vitamin D status in a subject, ${ }^{7} 1,25(\mathrm{OH}) 2 \mathrm{D} 3$ is the actual bioactive form of vitamin $\mathrm{D}$ that induces antimicrobial response, ${ }^{7}$ and so we also performed a meta-analysis on the association between 1,25(OH)2D3 and TB in order to determine whether there was any change of $1,25(\mathrm{OH}) 2 \mathrm{D} 3$ level in TB subjects compared to control without TB.

\section{Methods}

\section{Search strategy, inclusion and exclusion criteria, and data extraction}

PubMed/Medline and Web of Science (Web of Knowledge) databases were searched to identify all studies relating to vitamin D and TB using the following search terms: "vitamin D",
"VDD”, "hypovitaminosis D”, “tuberculosis", "TB”, "25-hydroxyvitamin D", and "1,25-dihydroxyvitamin D".

All properly controlled studies published in English on vitamin D and TB were potentially eligible for inclusion in our meta-analysis. We excluded reviews, meeting abstracts, and case-only studies.

Relevant data from each included study were extracted. Among the data extracted, relevant group meanvalues (standard deviations, SDs) were extracted from each included study whenever possible. When relevant values were presented only as median (interquartile range), mean (SD) was approximated as median (interquartile range/1.35); ${ }^{15}$ when relevant values were presented only as median (range), mean (SD) was approximated as median (range/4); ${ }^{16}$ when relevant values were presented only as mean values (standard errors, SEs), SD was extracted as SE $\times$ square root of sample size; ${ }^{17}$ and when relevant values were presented only as meanvalues (confidence intervals, CIs), SD was extracted as square root of sample size $\times$ (upper limit - lower limit) $/(2 \times Y)$, where $Y$ is the $t$ value from a size equal to sample size minus $1 .^{17}$

\section{Quality of the studies}

Each study's adequacy in three key areas (methodological, clinical, and statistical) was evaluated first. Then each study was screened with the Newcastle-Ottawa Scale. ${ }^{18}$

\section{Statistical analysis}

Cochrane Review Manager (RevMan 5.3, Copenhagen, Denmark, The Nordic Cochrane Centre) was used to perform all statistical analysis. Continuous outcomes were analyzed using mean difference (MD) or standardized MD (SMD), while dichotomous outcome was analyzed using pooled odds ratio (OR). Pooled OR and MD/SMD with their corresponding $95 \% \mathrm{CI}$ were calculated with a random-effect model because this model assumes a genuine diversity in the results caused by between-studies heterogeneity and incorporates a between-studies variance into the calculation accordingly. ${ }^{19}$ The $Z$ test was used to assess the statistical significance of the pooled OR and MD/SMD. Betweenstudies heterogeneity was assessed with the Cochran $Q$ statistic-based chi-square $\left(\chi^{2}\right)$ test, ${ }^{20}$ and $I^{2}$ index was the tool used to assess the heterogeneity, where a $I^{2}$ value around $25 \%, 50 \%$, and $75 \%$ indicated a low, moderate, and large heterogeneity, respectively. ${ }^{21}$ Finally, if no heterogeneity was found using the random-effect model $\left(I^{2}=0\right)$, data were analyzed again using a fixed-effect model. Statistical significance was considered with a $P$-value of $<0.05$, except for the $Q$ statistic where a $P$-value of $<0.10$ indicated statistically significance. 
Subpopulation analysis was performed according to the ethnicity of the subjects. In addition, funnel plots were used to evaluate the publication bias in each meta-analysis.

\section{Results}

\section{Eligible studies and characteristics of the studies}

Figure 1 illustrates our search process and results. From a total of 723 potentially eligible articles, 685 were excluded because they were irrelevant to our topic here, reviews, meeting abstracts, or case-only studies, or contained no detailed data needed for our meta-analysis. A total of 38 articles were included in our meta-analysis. ${ }^{1,2,4,5,11,12,14,22-52}$

\section{Vitamin D level was significantly lower in TB patients vs controls, and VDD was associated with an increased risk of TB}

A total of 25 studies with 3,599 TB cases and 3,063 control subjects were included in our analysis of serum/plasma 25(OH)D level in TB patients vs control (Figure 2A). ${ }^{1,4,5,11,12,14,22-28,30,35-44,46}$ Our analysis with a randomeffect model showed a significantly lower serum/plasma $25(\mathrm{OH}) \mathrm{D}$ level in TB patients vs controls ( $\mathrm{MD}=-13.05$; 95\% CI $\left.=[-19.02,-7.08] ; P<0.0001 ; I^{2}=96\right)$ (Figure 2A). Subgroup analysis showed similar result for the Asian population $(\mathrm{MD}=-14.64 ; 95 \% \mathrm{CI}=[-20.15,-9.13]$; $\left.P<0.00001 ; I^{2}=89\right)$; however, there was no such significant

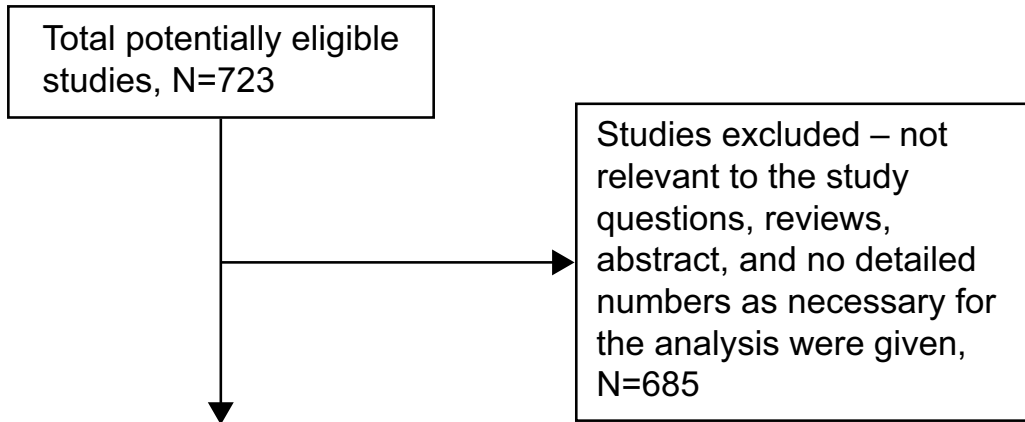

Studies included in the meta-analyses, $\mathrm{N}=38$

Studies included in the meta-analysis of serum/plasma 25-hydroxyvitamin $D(25(\mathrm{OH}) \mathrm{D})$ level in tuberculosis (TB) patients vs control, and of vitamin D deficiency (VDD) and TB, $N=21$, and 23, respectively.

Studies included in the meta-analysis of serum 25(OH)D level in active TB subject vs latent TB infection (LTBI) subjects/household contacts of active TB patients, of VDD and number of LTBI subjects or household contacts of TB patients developing active TB, and of VDD and risk of tuberculin skin test (TST) conversion/TB infection conversion (TBIC), $N=4,5$, and 3 , respectively.

Studies included in the meta-analysis of serum 25(OH)D level in HIV-TB coinfected patients vs HIV patients without TB, of VDD and risk of TB in HIV-infected patients, and of 25(OH)D level and risk of developing TB-associated immune reconstitution inflammatory syndrome (TB-IRIS) in HIV-TB coinfected patients receiving antiretroviral therapy (ART), $\mathrm{N}=4,4$, and 3 , respectively.

Studies included in the meta-analysis of the effect of 1-4 months of anti-TB treatment on 25(OH)D level in TB patients, of the effect of a full course of anti-TB treatment on 25(OH)D level in TB patients, and of $25(\mathrm{OH}) \mathrm{D}$ level in TB patients after anti-TB treatment vs control without TB, $N=3,4$, and 4 , respectively.

Studies included in the meta-analysis of the level of serum/plasma 1,25-dihydroxyvitamin D3 $(1,25(\mathrm{OH}) 2 \mathrm{D} 3)$ in TB patients vs control, $\mathrm{N}=4$ 


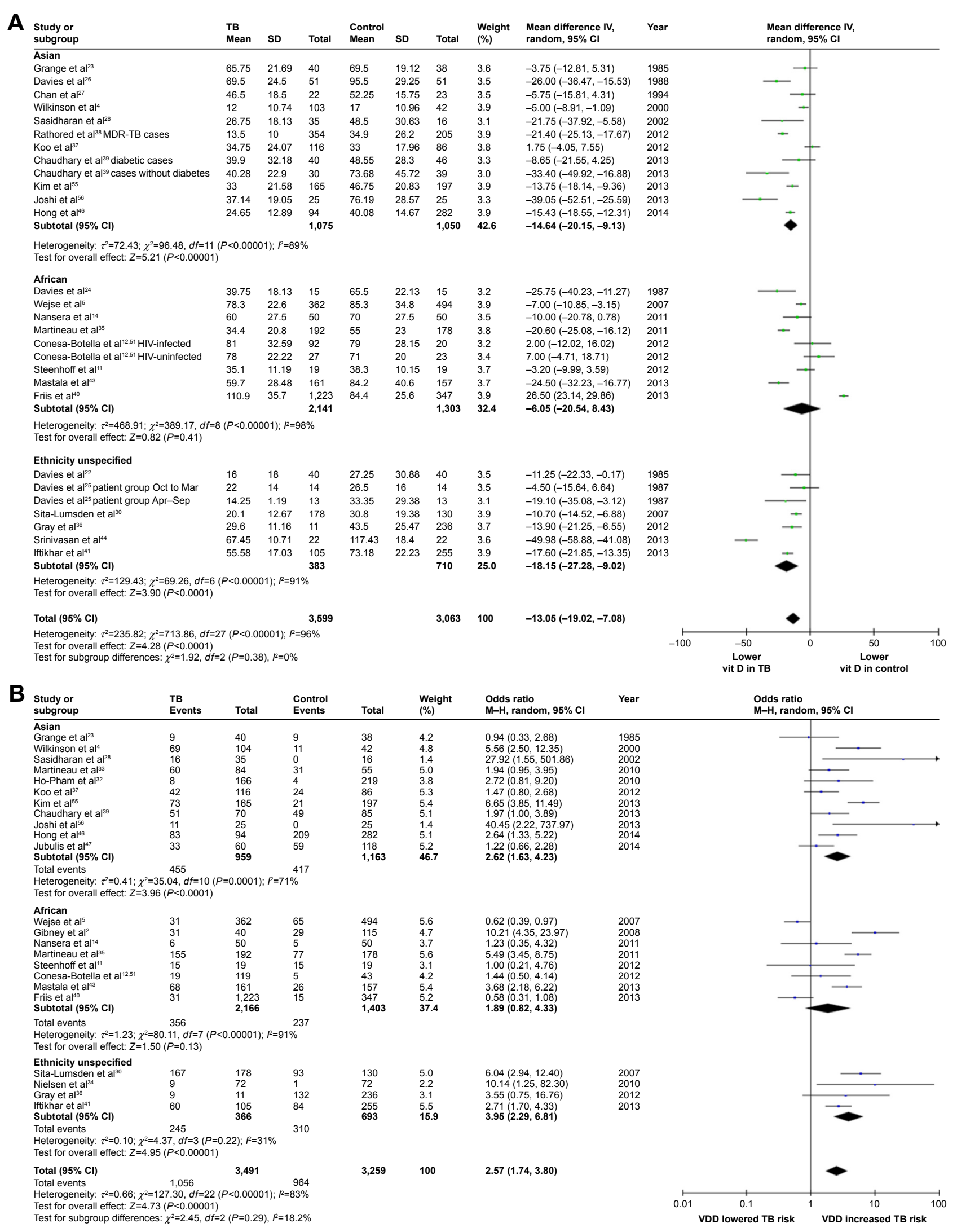

Figure 2 (A) Forest plot of comparison of vitamin D level (serum 25-hydroxyvitamin D [25(OH)D]) in TB patients vs control: overall effect for continuous outcome using a random-effect model. (B) Forest plot of association between VDD and risk of TB: overall effect for dichotomous outcome using a random-effect model. The diamonds stand for pooled effect.

Abbreviations: TB, tuberculosis; vit, vitamin; VDD, vitamin D deficiency; SD, standard deviation; Cl, confidence interval; df, degrees of freedom; IV, independent variable. 
difference for the African population ( $\mathrm{MD}=-6.05 ; 95 \%$ $\left.\mathrm{CI}=[-20.54,-8.43] ; P=0.41 ; P^{2}=98\right)($ Figure 2A). We found a similar result when the same analysis was performed excluding studies where cases and controls were subjects with other diseases such as HIV and diabetes, or where control subjects had LTBI (data not shown).

For our analysis on VDD and risk of TB, a total of 23 studies with 3,491 TB cases and 3,259 control subjects were included (Figure 2B) ${ }^{1,2,4,5,11,12,14,23,28,30,32-37,39,40-43,46,47}$ Our analysis showed that VDD was significantly associated with an increased risk of TB $(\mathrm{OR}=2.57 ; 95 \% \mathrm{CI}=[1.74,3.80]$; $P<0.00001 ; I^{2}=83$ ) (Figure 2B). Subgroup analysis revealed similar result for the Asian population $(\mathrm{OR}=2.62$; $\left.95 \% \mathrm{CI}=[1.63,4.23] ; P<0.0001 ; I^{2}=71\right)$; however, VDD was not associated with an increased risk of TB in the African population ( $\mathrm{OR}=1.89 ; 95 \% \mathrm{CI}=[0.82,4.33]$; $P=0.13 ; I^{2}=91$ ) (Figure 2B). Results were similar when the same analysis was performed excluding studies where cases and controls were subjects with other diseases such as HIV and diabetes, or where control subjects had LTBI (data not shown).

\section{VDD was more likely a risk factor for TB than its consequence}

Four studies with 331 active TB cases and 326 LTBI subjects/ household contacts of active TB patients were included in our meta-analysis (Figure 3A).,35,36,42 We included household contacts of active TB patients as part of our control in this analysis because they were at a high risk of becoming infected and developing $\mathrm{TB},{ }^{42}$ and a meta-analysis by Fox et $\mathrm{al}^{53}$ reported a high prevalence of LTBI in contacts of active TB patients $(51.5 \%$; 95\% CI $=[47.1 \%, 55.8 \%]$; $I^{2}=98.9 \%$ ). Our analysis revealed a trend toward a lower serum/plasma 25(OH)D level in active TB patients vs LTBI subjects/household contacts of active TB patients; however, this trend did not reach the level of statistical

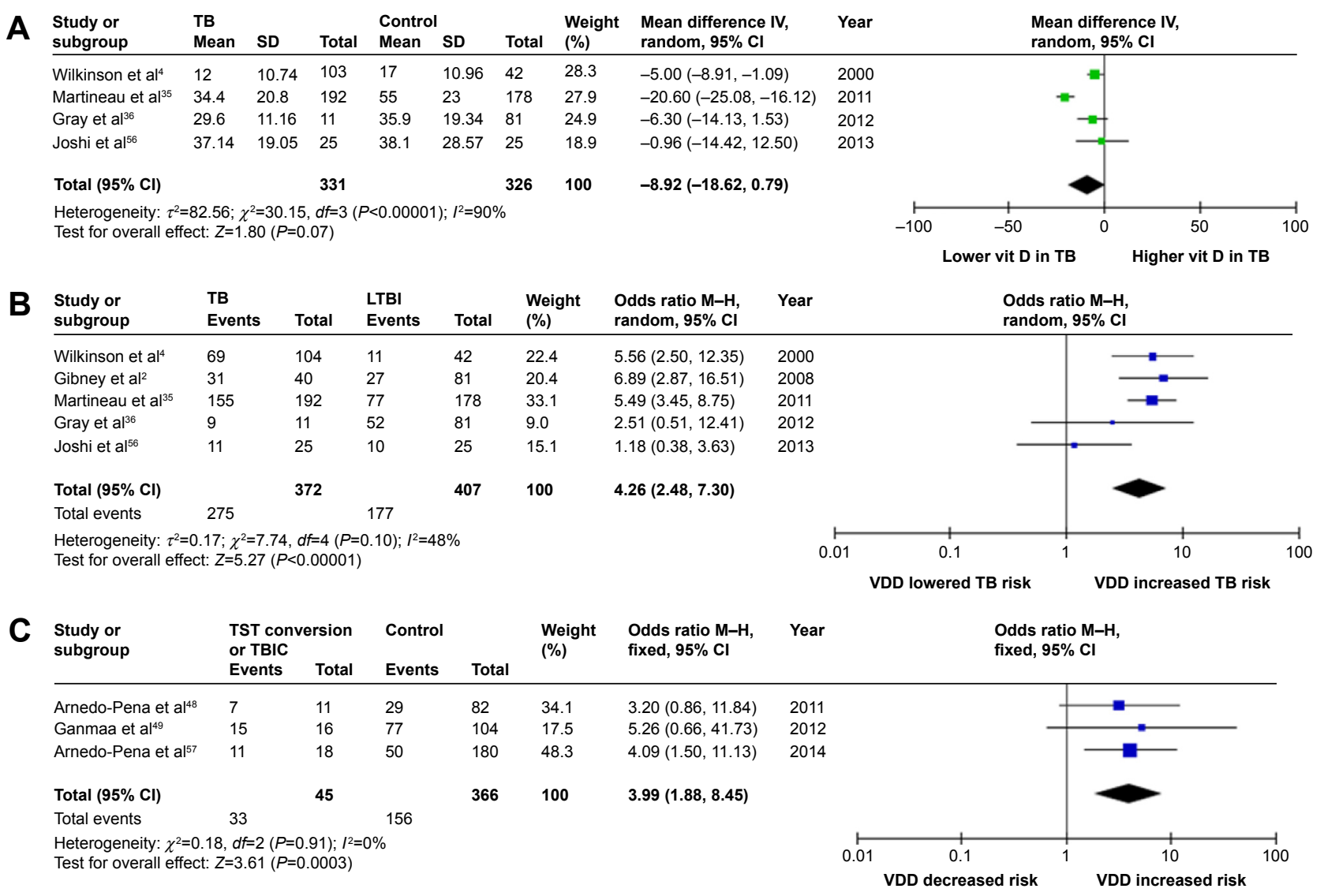

Figure 3 (A) Forest plot of comparison of serum 25(OH)D level in active TB patients vs LTBI subjects/household contacts of active TB patients: overall effect for continuous outcome using a random-effect model. (B) Forest plot of association between VDD and risk of developing active TB in LTBI subjects or household contacts of TB patients: overall effect for dichotomous outcome using a random-effect model. (C) Forest plot of association between VDD and risk of TST conversion/TBIC: overall effect for dichotomous outcome using a fixed-effect model. The diamonds stand for pooled effect.

Abbreviations: TB, tuberculosis; LTBI, latent TB infection; vit, vitamin; VDD, vitamin D deficiency; TST, tuberculin skin test; TBIC, TB infection conversion; SD, standard deviation; $\mathrm{Cl}$, confidence interval; $d f$, degrees of freedom; IV, independent variable. 
significance $(\mathrm{MD}=-8.92 ; 95 \% \mathrm{CI}=[-18.62,0.79] ; P=0.07$; $\left.r^{2}=90\right)$ (Figure 3A).

\section{VDD was significantly associated with an increased} risk of developing active TB in LTBI subjects/

\section{household contacts of TB patients}

Five studies with 372 active TB patients and 407 LTBI subjects/household contacts of active TB patients were included in our analysis (Figure 3B)., 2,435,36,42 VDD was positively and significantly associated with an increased risk of developing active TB in LTBI subjects/household contacts of active TB patients ( $\mathrm{OR}=4.26 ; 95 \% \mathrm{CI}=[2.48,7.30]$; $P<0.00001 ; P^{2}=48 \%$ ) (Figure 3B).

\section{VDD was significantly associated with an increased} risk of TST conversion/TBIC

Our meta-analysis here included three studies with 45 cases with TST conversion/TBIC and 366 control subjects without
TST conversion/TBIC (Figure 3C)..$^{4-50}$ Our analysis using a fixed-effect model revealed significant association between VDD and an increased risk of TST conversion/ TBIC $(\mathrm{OR}=3.99 ; 95 \% \mathrm{CI}=[1.88,8.45] ; P=0.0003$; $r^{2}=0$ ) (Figure 3C).

\section{Vitamin D, TB, and HIV}

No significant vitamin D level difference in HIV-TBcoinfected African patients vs African HIV patients without active TB, and VDD was not associated with an increased risk of TB in African HIV-infected patients

Our meta-analysis comparing vitamin D level in HIVTB-coinfected patients vs HIV patients without active TB included four studies on the African population with 260 HIV-TB-coinfected cases and 164 control HIV subjects without active TB (Figure 4A). ${ }^{11,12,14,35}$ Serum/plasma 25(OH)D level in HIV-TB-coinfected African patients was not

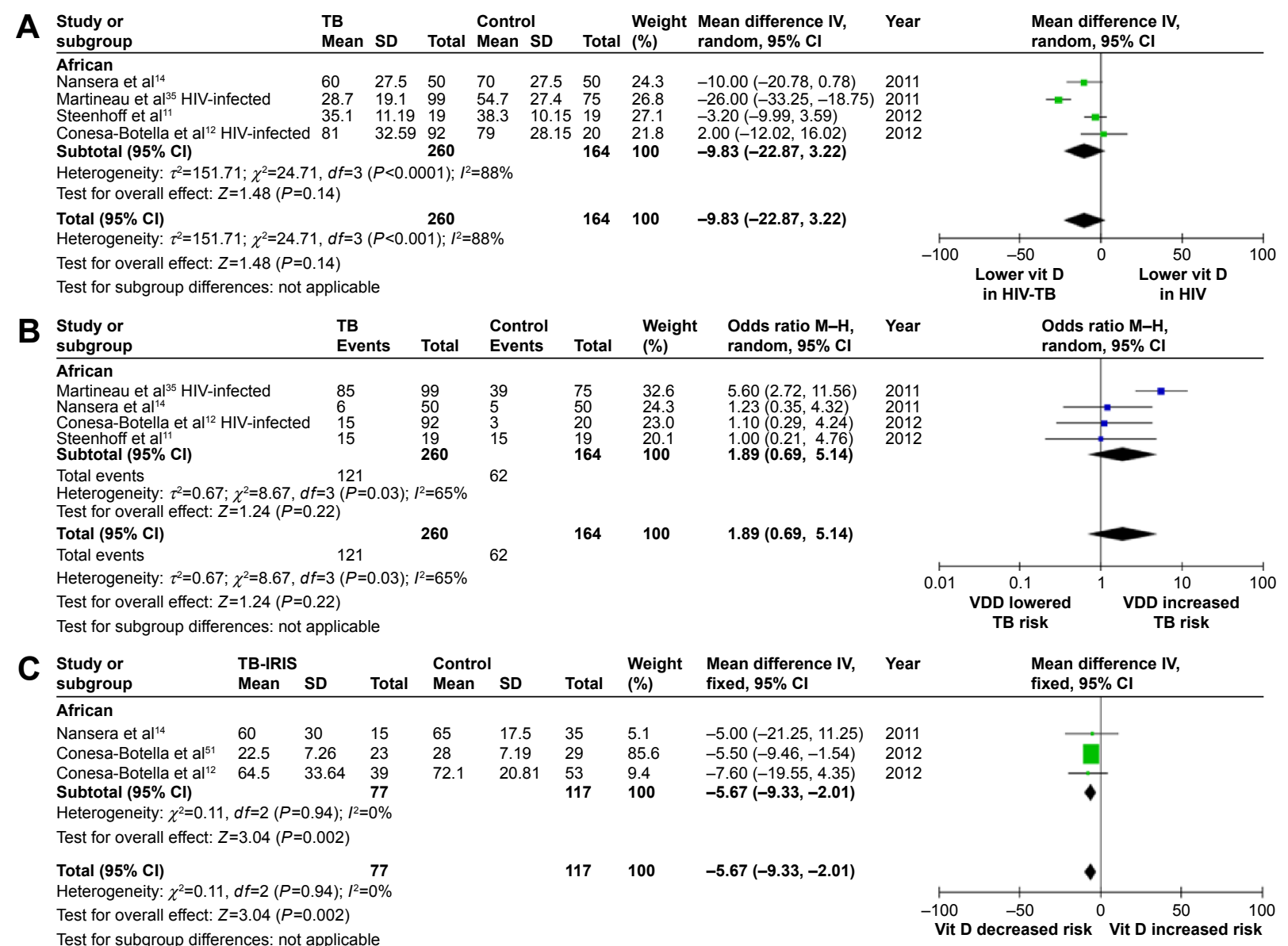

Figure 4 (A) Forest plot of comparison of serum 25(OH)D level in HIV-TB-coinfected patients vs HIV patients without active TB: overall effect for continuous outcome using a random-effect model. (B) Forest plot of association between VDD and risk of TB in HIV-infected patients: overall effect for dichotomous outcome using a randomeffect model. (C) Forest plot comparing 25(OH)D level in HIV-TB-coinfected patients receiving ART who developed TB-IRIS vs HIV-TB-coinfected patients receiving ART who did not develop TB-IRIS: overall effect for continuous outcome using a fixed-effect model. The diamonds stand for pooled effect.

Abbreviations: HIV, human immunodeficiency virus; TB, tuberculosis; ART, antiretroviral therapy; TB-IRIS, TB-associated immune reconstitution inflammatory syndrome; vit, vitamin; VDD, vitamin D deficiency; SD, standard deviation; Cl, confidence interval; $d f$, degrees of freedom; IV, independent variable. 
significantly different from $\mathrm{HIV}$-infected African patients without active TB $(\mathrm{MD}=-9.83 ; 95 \% \mathrm{CI}=[-22.87,3.22]$; $P=0.14 ; I^{2}=88$ ) (Figure 4A).

The same four studies on African population with 260 HIV-TB-coinfected cases and 164 control HIV subjects without active TB were included in our analysis on the association between VDD and risk of TB in HIV-infected patients (Figure 4B). ${ }^{11,12,14,35}$ There was no significant association between VDD and risk of TB in African HIVinfected patients $(\mathrm{OR}=1.89 ; 95 \% \mathrm{CI}=[0.69,5.14] ; P=0.22$; $\left.I^{2}=65\right)$ (Figure 4B).

Significantly lower vitamin D level in HIV-TBcoinfected African patients receiving ART who developed TB-IRIS vs those who did not develop TB-IRIS

Three studies with 77 HIV-TB-coinfected African cases receiving ART who developed TB-IRIS and 117 African control patients who did not develop TB-IRIS were included in this analysis (Figure 4C). ${ }^{12,14,51}$ HIV-TB-coinfected African cases receiving ART who developed TB-IRIS had a significantly lower vitamin D level vs those who did not develop TB-IRIS (MD $=-5.67 ; 95 \% \mathrm{CI}=[-9.33,-2.01]$; $\left.P=0.002 ; I^{2}=0\right)$ (Figure 4C).

\section{Anti-TB treatment and vitamin D level}

\section{Anti-TB treatment did not affect vitamin $D$ level in} TB patients receiving the treatment

Three studies with 224 TB patients receiving 1-4 months of anti-TB treatments ${ }^{25,37,52}$ and four studies with 391 TB patients receiving full course of anti-TB treatment ${ }^{1,25,37,46}$ were included in our analysis on whether anti-TB treatment affected vitamin D level in TB patients receiving the treatment (Figure 5A and B). Neither 1-4 months of anti-TB treatment $\left(\mathrm{MD}=1.47 ; 95 \% \mathrm{CI}=[-2.79,5.73] ; P=0.50 ; P^{2}=57\right)$ (Figure 5A) nor a full course of anti-TB treatment $(\mathrm{MD}=5.03$; $\left.95 \% \mathrm{CI}=[-3.20,13.25] ; P=0.23 ; I^{2}=86\right)$ (Figure 5B) affected $25(\mathrm{OH}) \mathrm{D}$ level significantly.

\section{TB patients still had a significantly lower vitamin D level after completion of anti-TB treatment than controls without TB}

Four studies with 327 TB cases after completion of anti-TB treatment and 592 control subjects without TB were included (Figure 5C). ${ }^{1,25,37,46}$ Vitamin D level was still significantly lower in TB patients after completion of their anti-TB treatment vs control subjects without TB (MD $=-8.05 ; 95 \% \mathrm{CI}=[-13.56,-2.54] ; P=0.004$; $\left.I^{2}=68\right)$ (Figure $5 \mathrm{C}$ ), especially for the Asian population
$(\mathrm{MD}=-9.36 ; 95 \% \mathrm{CI}=[-15.23,-3.48] ; P=0.002 ;$ $I^{2}=80$ ) (Figure 5C).

\section{A trend of higher I,25(OH)2D3 level in TB patients vs controls without TB}

Although serum 25(OH)D is the commonly used measure of vitamin D status in a subject, ${ }^{7} 1,25(\mathrm{OH}) 2 \mathrm{D} 3$ is the actual bioactive form of vitamin $\mathrm{D}$ that induces antimicrobial response, ${ }^{7}$ and therefore we performed a meta-analysis comparing 1,25(OH)2D3 level in TB subjects vs controls without TB. Four studies with 160 TB cases and 338 control subjects without TB were included (Figure 6). ${ }^{22,29,31,45}$ There was a trend toward higher $1,25(\mathrm{OH}) 2 \mathrm{D} 3$ level in TB patients vs controls; however, this trend did not reach the level of statistical significance $(\mathrm{SMD}=1.02 ; 95 \% \mathrm{CI}=[-0.09,2.14]$; $P=0.07 ; I^{2}=95$ ) (Figure 6).

\section{Between-studies heterogeneity and publication bias}

Between-studies heterogeneity varied from none to high for our meta-analyses with $I^{2}$ values ranging from 0 to 96 (Figures 2A and B, 3A-C, 4A-C, 5A-C, and 6). Furthermore, funnel plots for all of our meta-analyses were symmetrical, suggesting the presence of none or very little publication bias in our analyses (data not shown).

\section{Discussion}

Our meta-analysis on the association between vitamin D and TB showed a significantly lower level of vitamin D in TB patients vs controls and that VDD was positively associated with an increased risk of TB, which is consistent with the results of the meta-analysis by Nnoaham et al. ${ }^{13}$ In the subgroup analysis, although we found similar results for the Asian population, such an association was lacking in the African population even when we excluded studies where cases and controls had other diseases such as HIV and diabetes, or where the control subjects had LTBI. This suggested the possibility of ethnic difference in the role of vitamin D in TB that warrants further investigation. As sunlight and diet are two major sources of vitamin D for humans, ${ }^{13}$ adequate exposure to sunlight and/or adjustment of diet to maintain proper vitamin $\mathrm{D}$ level in our bodies is be deemed a necessity.

As HIV-infected subjects are more susceptible to TB and have greater mortality and morbidity caused by $\mathrm{TB},{ }^{14}$ in order to further clarify whether vitamin D played any role in occurrence and deterioration of TB in HIV-infected subjects, we also analyzed the association between vitamin D level/ VDD and TB in HIV-infected subjects and the association 


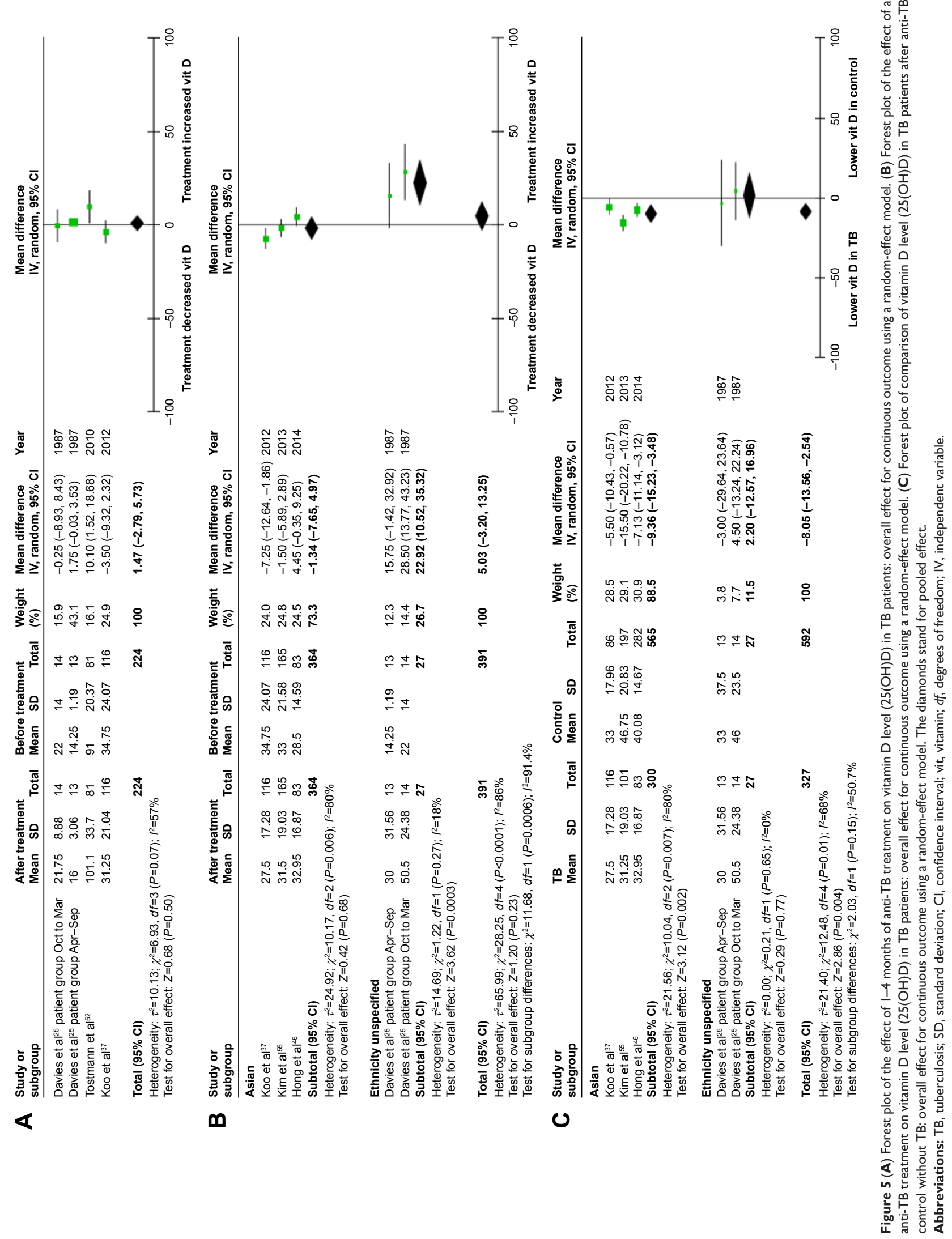




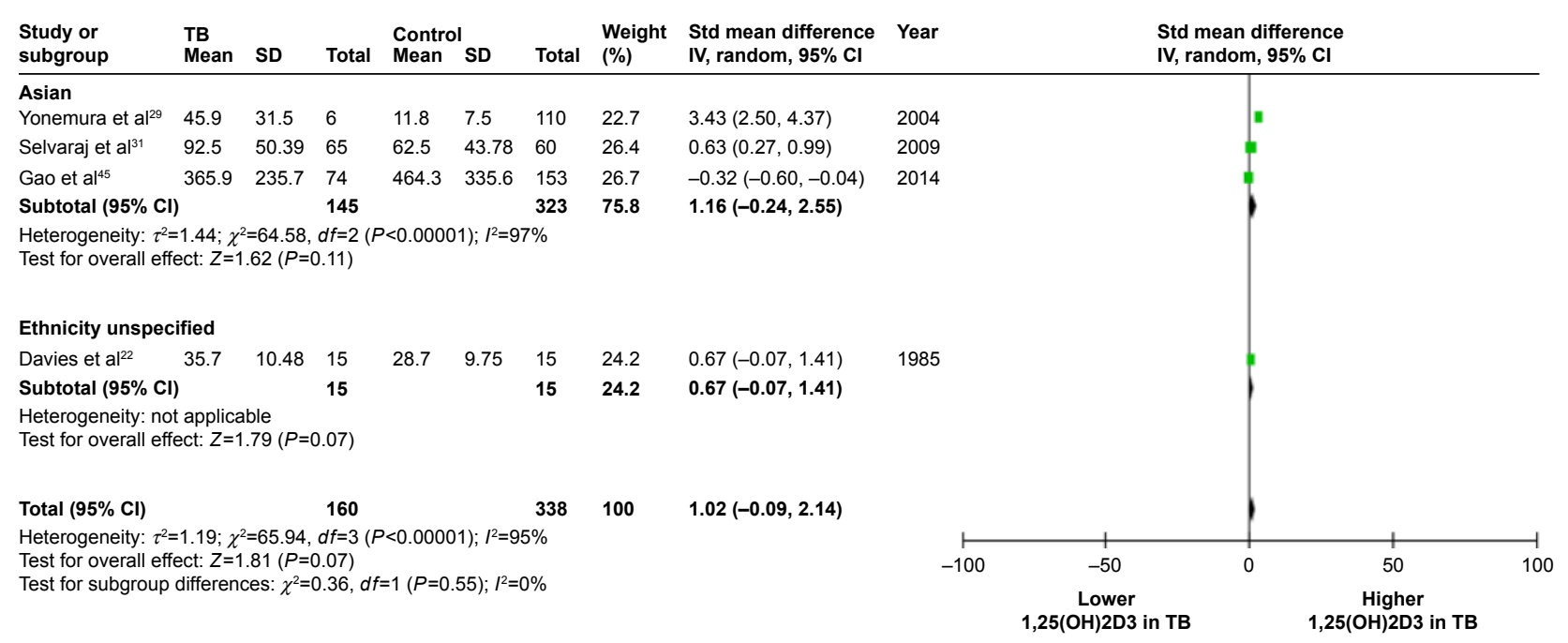

Figure 6 Forest plot of comparison of the level of the vitamin D metabolite - serum/plasma I,25-dihydroxyvitamin D3 (I,25(OH)2D3) in TB patients vs control without TB: overall effect for continuous outcome using a random-effect model.

Note: The diamonds stand for pooled effect.

Abbreviations: TB, tuberculosis; SD, standard deviation; Cl, confidence interval; df, degrees of freedom; IV, independent variable.

between vitamin D level and risk of developing TB-IRIS in HIV-TB-coinfected patients receiving ART. Our analysis showed that there was no significant difference in vitamin $\mathrm{D}$ level in HIV-TB-coinfected African patients vs African HIV patients without active TB and that VDD was not associated with an increased risk of TB in African HIV-infected patients. However, a significantly lower vitamin D level was found in HIV-TB-coinfected African patients receiving ART who developed TB-IRIS vs those who did not. In view of the fact that our analysis showed lack of association between vitamin $\mathrm{D}$ and TB in the African population, such lack of association between vitamin D and TB in the HIV-infected African population should be interpreted with caution as to whether it represents a true lack of association in HIV-infected subjects; studies on HIV-infected subjects of other ethnicities are needed to confirm these results. However, if such a lack of association is indeed true, Steenholf et $\mathrm{al}^{11}$ suggested that as HIV-infected subjects are already immunocompromised, it is possible that any immune response induced by vitamin $\mathrm{D}$ would also be compromised, thus masking any role vitamin $\mathrm{D}$ played in fighting TB; this, along their observation that HIV-infected subjects without TB were already plagued with VDD, could provide a possible explanation for such a lack of association between vitamin D and TB in HIV-infected subjects. On the other hand, our finding that HIV-TB-coinfected subjects who developed TB-IRIS (a paradoxical worsening of TB after initial improvement upon TB treatment after ART initiation or manifestation of TB clinically not apparent before ART initiation) had a lower vitamin D level that those who did not suggest that vitamin D was somehow still involved in TB occurrence and progression in HIV-infected patients. Conesa-Botella et a ${ }^{51}$ reported an association between VDD and elevated proinflammatory cytokine and that corticosteroid therapy could modify patients' inflammatory profile and possibly even reduce the severity of TB-IRIS symptom and suggested vitamin D supplement prior to ART initiation to determine whether it decreased TB-IRIS incidence. Meanwhile, as only a limited number of studies with limited sample sizes were included in our analyses, more studies with larger sample sizes are needed to further elucidate whether there is any association between VDD and TB/TB-IRIS.

Also, our analysis found a significant association between VDD and TB, but it did not tell us whether VDD was a risk factor or a consequence of TB; however, our analysis revealed that VDD was significantly associated with an increased risk of developing active TB in LTBI subjects/household contacts of TB patients, that VDD was significantly associated with an increased risk of TST conversion/TBIC, and that the trend toward a lower vitamin $\mathrm{D}$ level in active TB patients vs LTBI subjects/household contacts of active TB patients did not reach statistical significance. These results suggested that VDD was more likely a risk factor for TB rather than its consequence. Had VDD been a manifestation of malnutrition generally associated with TB, we would expect a more significant difference in vitamin $\mathrm{D}$ level between active TB patients vs LTBI subjects/household contacts of active TB patients and VDD would not have been significantly associated with an increased risk of LTBI developing into active TB or TST conversion/TBIC. On the contrary, our result suggested that vitamin D was possibly involved in 
the early stage of TB infection and symptom manifestation; in addition, it has been shown that 1,25(OH)2D3 activates VDR signaling and induces antimicrobial responses such as induction of autophagy, phagolysosomal fusion, release and activation of the antimicrobial peptide cathelicidin, and killing of intracellular M. tuberculosis. ${ }^{7-9}$ All of these results pointed to the fact that VDD is more likely a risk factor for TB. This possibility was further strengthened by our results that anti-TB treatment did not significantly affect vitamin $\mathrm{D}$ level in TB patients receiving the treatment and that TB subjects after completion of anti-TB treatment still had a significantly lower vitamin D level than control subjects without TB. This was because if VDD had been a consequence of TB, we would expect a significantly elevated vitamin D level in TB subjects after effective anti-TB treatment. Overall, our analyses indicated that VDD was most likely a risk factor for TB.

Since our analysis indicated that VDD was most likely a risk factor for TB, this raises a question of whether vitamin $\mathrm{D}$ supplement would be beneficial to TB prevention and treatment. The meta-analysis by Xia et $\mathrm{al}^{54}$ in 2012 reported that vitamin D supplementation did not show a significant benefit to TB treatment; however, the meta-analysis did not address the question of whether it would be beneficial to TB prevention. Rigorously controlled studies will be needed to further determine whether vitamin D supplementation would be beneficial.

Because 1,25(OH)D3 is the bioactive form of vitamin $\mathrm{D},{ }^{7}$ we also analyzed the level of $1,25(\mathrm{OH}) 2 \mathrm{D} 3$ in TB subjects vs controls and found a trend of higher 1,25(OH)2D3 level in TB patients vs controls without TB. Selvaraj et $\mathrm{al}^{31}$ postulated that this possible increase in 1,25(OH)2D3 could be because of CYP27B1 expression upregulation that led to increased conversion of $25(\mathrm{OH}) \mathrm{D}$ to $1,25(\mathrm{OH}) 2 \mathrm{D} 3$, which could then cause $25(\mathrm{OH}) \mathrm{D}$ deficiency because of its increased use. However, if this is the case, it would mean that VDD would be a consequence of TB rather than one of its causes and would be inconsistent with our previous conclusions. On the other hand, as only four studies with limited sizes were included in our analysis, this result is far from certain, and more studies are needed to confirm or refute this result.

There are certain strengths and limitations in our study. Its strength lies in the fact that it was a comprehensive analysis of multiple aspects of the relationship between vitamin $\mathrm{D}$ and $\mathrm{TB}$ and helped to answer the question of whether vitamin $D$ was one of the causes or consequences of TB, and our analysis included all relevant available studies of reasonable quality to ensure that the resulting picture was as complete as possible. However, our study also has certain limitations. For some of our analyses, the number of relevant studies was limited and their sample sizes were small, which undoubtedly would affect the certainty of some of our results. Furthermore, because different studies used different standards for VDD, such inconsistency in VDD definition would also affect our results.

\section{Conclusion}

Our analyses revealed a significantly lower vitamin D level in TB subjects and that VDD was associated with more risk of TB. VDD is more likely a risk factor for TB than its consequence, and more studies are needed to determine whether vitamin D supplement is beneficial to TB prevention and treatment.

\section{Disclosure}

The authors report no conflicts of interest in this work.

\section{References}

1. Kim JH, Park JS, Cho YJ, et al. Low serum 25-hydroxyvitamin D level: an independent risk factor for tuberculosis? Clin Nutr. 2014;33(6): 1081-1086.

2. Gibney KB, MacGregor L, Leder K, et al. Vitamin D deficiencyis associated with tuberculosis and latent tuberculosis infection in immigrants from sub-Saharan Africa. Clin Infect Dis. 2008;46(3):443-446.

3. Chocano-Bedoya P, Ronnenberg AG. Vitamin D and tuberculosis. Nutr Rev. 2009;67(5):289-293.

4. Wilkinson RJ, Llewelyn M, Toossi Z, et al. Influence of vitamin D deficiency and vitamin D receptor polymorphisms on tuberculosis among Gujarati Asians in West London: a case-control study. Lancet. 2000; 355(9204):618-621.

5. Wejse C, Olesen R, Rabna P, et al. Serum 25-hydroxyvitamin D in a West African population of tuberculosis patients and unmatched healthy controls. Am J Clin Nutr. 2007;86(5):1376-1383.

6. Ustianowski A, Shaffer R, Collin S, Wilkinson RJ, Davidson RN. Prevalence and associations of vitamin $\mathrm{D}$ deficiency in foreign-born persons with tuberculosis in London. $J$ Infect. 2005;50(5):432-437.

7. Realegeno S, Modlin RL. Shedding light on the vitamin D-tuberculosisHIV connection. Proc Natl Acad Sci U S A. 2011;108:18861-18862.

8. Liu PT, Stenger S, Li H, et al. Toll-like receptors triggering of a vitamin D mediated anti-microbical response. Science. 2006;311(5768): 1770-1773.

9. Adams JS, Ren S, Liu PT, et al. Vitamin D directed rheostatic regulation of monocyte antibacterial responses. J Immunol. 2009;182(7): 4289-4295.

10. Friis H, Range N, Pedersen ML, et al. Hypovitaminosis D is common among pulmonary tuberculosis patients in Tanzania but is not explained by the acute phase response. $J$ Nutr. 2008;138(12):2474-2480.

11. Steenhoff AP, Redwood A, Pettifor JM, et al. Vitamin D status in HIVinfected patients with and without tuberculosis: a pilot study. J Acquir Immune Defic Syndr. 2012;61(2):e21-e23.

12. Conesa-Botella A, Goovaerts O, Massinga-Loembé M, et al. Low prevalence of vitamin D deficiency in Ugandan HIV-infected patients with and without tuberculosis. Int J Tuberc Lung Dis. 2012;16(11): 1517-1521.

13. Nnoaham KE, Clarke A. Low serum vitamin D levels and tuberculosis: a systematic review and meta-analysis. Int J Epidemiol. 2008; 37:(1)113-119. 
14. Nansera D, Graziano FM, Friedman DJ, Bobbs MK, Jones AN, Hansen KE. Vitamin D and calcium levels in Ugandan adults with human immunodeficiency virus and tuberculosis. Int J Tuberc Lung Dis. 2011; 15(11):1522-1527.

15. Higgins JP, Green S. Cochrane Handbook for Systematic Reviews of Interventions. Version 5.1.0 [updated March 2011], Section 7.7.3.5 Medians and interquartile ranges. The Cochrane Collaboration, 2011. Available from: http://handbook.cochrane.org/

16. Higgins JP, Green S. Cochrane Handbook for Systematic Reviews of Interventions. Version 5.1.0 [updated March 2011], Section 7.7.3.6 Ranges. The Cochrane Collaboration, 2011. Available from: http:// handbook.cochrane.org/

17. Higgins JP, Green S. Cochrane Handbook for Systematic Reviews of Interventions. Version 5.1.0 [updated March 2011], Section 7.7.3.2 Obtaining standard deviations from standard errors and confidence intervals for group means. The Cochrane Collaboration, 2011. Available from: http://handbook.cochrane.org/

18. Wells GA, Shea B, O'Connell D, et al. The Newcastle-Ottawa Scale (NOS) for assessing the quality of non-randomised studies in metaanalysis. Ottawa, ON: Ottawa Health Research Institute; 2004.

19. DerSimonian R, Laird N. Meta-analysis in clinical trials. Control Clin Trials. 1986;7:177-188.

20. Higgins J, Thompson S, Deeks J, Altman D. Statistical heterogeneity in systematic reviews of clincial trials: a critical appraisal of guidelines and practice. J Health Serv Res Policy. 2002;7(1):51-61.

21. Navarro-Mateu F, Escámez T, Koenen KC, Alonso J, Sánchez-Meca J. Meta-analyses of the 5-HTTLPR polymorphism and post-traumatic stress disorder. PLoS One. 2013;8(6):e66227.

22. Davies PD, Brown RC, Woodhead JS. Serum concentrations of vitamin D metabolites in untreated tuberculosis. Thorax. 1985;40(8):187-190.

23. Grange JM, Davies PD, Brown RC, Woodhead JS, Kardjito T. A study of vitamin D levels in Indonesian patients with untreated pulmonary tuberculosis. Tubercle. 1985;66(3):187-191.

24. Davies PD, Church HA, Brown RC, Woodhead JS. Raised serum calcium in tuberculosis patients in Africa. Eur J Respir Dis. 1987;71(5): 341-344.

25. Davies PD, Brown RC, Church HA, Woodhead JS. The effect of antituberculosis chemotherapy on vitamin D and calcium metabolism. Tubercle. 1987;68(4):261-266.

26. Davies PD, Church HA, Bovornkitti S, Charumilind A, Byrachandra S. Altered vitamin D homeostasis in tuberculosis. Int Med Thailand. 1988;4:45-47.

27. Chan TY, Poon P, Pang J, et al. A study of calcium and vitamin D metabolism in Chinese patients with pulmonary tuberculosis. J Trop Med Hyg. 1994;97(1):26-30.

28. Sasidharan PK, Rajeev E, Vijayakumari V. Tuberculosis and vitamin D deficiency. J Assoc Physicians India. 2002;50:554-558.

29. Yonemura K, Ohtake T, Matsushima H, Fujigaki Y, Hishida A. High ratio of 1,25-dihydroxyvitamin D3 to parathyroid hormone in serum of tuberculous patients with end-stage renal disease. Clin Nephrol. 2004;62(3):202-207.

30. Sita-Lumsden A, Lapthorn G, Swaminathan R, Milburn HJ. Reactivation of tuberculosis and vitamin $\mathrm{D}$ deficiency: the contribution of diet and exposure to sunlight. Thorax. 2007;62(11):1003-1007.

31. Selvaraj P, Prabhu Anand S, Harishankar M, Alagarasu K. Plasma 1,25 dihydroxy vitamin D3 level and expression of vitamin d receptor and cathelicidin in pulmonary tuberculosis. J Clin Immunol. 2009;29(4):470-478.

32. Ho-Pham LT, Nguyen ND, Nguyen TT, et al. Association between vitamin D insufficiency and tuberculosis in a Vietnamese population. BMC Infect Dis. 2010;10:306.

33. Martineau AR, Leandro AC, Anderson ST, et al. Association between Gc genotype and susceptibility to TB is dependent on vitamin D status. Eur Respir J. 2010;35(5):1106-1112.

34. Nielsen NO, Skifte T, Andersson M, et al. Both high and low serum vitamin $\mathrm{D}$ concentrations are associated with tuberculosis: a case-control study in Greenland. Br J Nutr. 2010;104(10):1487-1491.
35. Martineau AR, Nhamoyebonde S, Oni T, et al. Reciprocal seasonal variation in vitamin D status and tuberculosis notifications in Cape Town, South Africa. Proc Natl Acad Sci U S A. 2011;108(47):19013-19017.

36. Gray K, Wood N, Gunasekera H, et al. Vitamin D and tuberculosis status in refugee children. Pediatr Infect Dis J. 2012;31(5):521-523.

37. Koo HK, Lee JS, Jeong YJ, et al. Vitamin D deficiency and changes in serum vitamin D levels with treatment among tuberculosis patients in South Korea. Respirology. 2012;17(5):808-813.

38. Rathored J, Sharma SK, Singh B, et al. Risk and outcome of multidrugresistant tuberculosis: vitamin D receptor polymorphisms and serum 25(OH)D. Int J Tuberc Lung Dis. 2012;16(11):1522-1528.

39. Chaudhary S, Thukral A, Tiwari S, Pratyush DD, Singh SK. Vitamin D status of patients with type 2 diabetes and sputum positive pulmonary tuberculosis. Indian J Endocrinol Metab. 2013;17(Suppl 3):S670-S673.

40. Friis H, Range N, Changalucha J, et al. Vitamin D status among pulmonary TB patients and non-TB controls: a cross-sectional study from Mwanza, Tanzania. PLoS One. 2013;8(12):e81142.

41. Iftikhar R, Kamran SM, Qadir A, Haider E, Bin Usman H. Vitamin D deficiency in patients with tuberculosis. J Coll Physicians Surg Pak. 2013;23(10):780-783.

42. Joshi L, Ponnana M, Penmetsa SR, Nallari P, Valluri V, Gaddam S. Serum vitamin D levels and VDR polymorphisms (BsmI and FokI) in patients and their household contacts susceptible to tuberculosis. Scand J Immunol. 2014;79(2):113-119.

43. Mastala Y, Nyangulu P, Banda RV, Mhemedi B, White SA, Allain TJ. Vitamin D deficiency in medical patients at a central hospital in Malawi: a comparison with TB patients from a previous study. PLoS One. 2013; 8(3):e59017.

44. Srinivasan A, Syal K, Banerjee D, et al. Low plasma levels of cholecalciferol and 13-cis-retinoic acid in tuberculosis: implications in host-based chemotherapy. Nutrition. 2013;29(10):1245-1251.

45. Gao WW, Wang Y, Zhang XR, et al. Levels of 1,25(OH)2D3 for patients with pulmonary tuberculosis and correlations of $1,25(\mathrm{OH}) 2 \mathrm{D} 3$ with the clinical features of TB. J Thorac Dis. 2014;6(6):760-764.

46. Hong JY, Kim SY, Chung KS, et al. Association between vitamin D deficiency and tuberculosis in a Korean population. Int J Tuberc Lung Dis. 2014;18(1):73-78.

47. Jubulis J, Kinikar A, Ithape M, et al. Modifiable risk factors associated with tuberculosis disease in children in Pune, India. Int J Tuberc Lung Dis. 2014;18(2):198-204.

48. Arnedo-Pena A, Juan-Cerdán JV, Romeu-Garcia A, et al. Latent tuberculosis infection, tuberculin skin test and vitamin D status in contacts of tuberculosis patients: a cross-sectional and case-control study. $B M C$ Infect Dis. 2011;11:349.

49. Ganmaa D, Giovannucci E, Bloom BR, et al. Vitamin D, tuberculin skin test conversion, and latent tuberculosis in Mongolian school-age children: a randomized, double-blind, placebo-controlled feasibility trial. Am J Clin Nutr. 2012;96(2):391-396.

50. Arnedo-Pena A, Juan-Cerdán JV, Romeu-García MA, et al. Vitamin D status and incidence of tuberculosis infection conversion in contacts of pulmonary tuberculosis patients: a prospective cohort study. Epidemiol Infect. 2015;143(8):1731-1741.

51. Conesa-Botella A, Meintjes G, Coussens AK, et al. Corticosteroid therapy, vitamin D status, and inflammatory cytokine profile in the HIV-tuberculosis immune reconstitution inflammatory syndrome. Clin Infect Dis. 2012;55(7):1004-1011.

52. Tostmann A, Wielders JP, Kibiki GS, Verhoef H, Boeree MJ, van der Ven AJ. Serum 25-hydroxy-vitamin D3 concentrations increase during tuberculosis treatment in Tanzania. Int J Tuberc Lung Dis. 2010;14(9): 1147-1152.

53. Fox GJ, Barry SE, Britton WJ, Marks GB. Contact investigation for tuberculosis: a systematic review and meta-analysis. Eur Respir J. 2013; 41(1):140-156.

54. Xia J, Shi L, Zhao L, Xu F. Impact of vitamin D supplementation on the outcome of tuberculosis treatment: a systematic review and meta-analysis of randomized controlled trials. Chin Med J (Engl). 2014;127(17): 3127-3134. 
55. Kim JH, Park JS, ChoYJ, et al. Low serum 25-hydroxyvitamin D level: an independent risk factor for tuberculosis? Clin Nutr. 2014;33(6): 1081-1086 (Epub 2013 Nov 26).

56. Joshi L, Ponnana M, Penmetsa SR, Nallari P, Valluri V, Gaddam S. Serum vitamin D levels and VDR polymorphisms (BsmI and FokI) in patients and their household contacts susceptible to tuberculosis. Sand J Immunol. 2014;79:113-119 (Epub 2013).
57. Arnedo-Pena A, Juan Cerdan JV, Romeu-Garcia A, et al. Latent tuberculosis infection, tuberculin skin test and vitamin D status in contacts of tuberculosis patients: a cross-sectional and case-control study. BMC Infect Dis. 2011;11:349.

\section{Publish your work in this journal}

Drug Design, Development and Therapy is an international, peerreviewed open-access journal that spans the spectrum of drug design and development through to clinical applications. Clinical outcomes, patient safety, and programs for the development and effective, safe, and sustained use of medicines are the features of the journal, which has also been accepted for indexing on PubMed Central. The manuscript management system is completely online and includes a very quick and fair peer-review system, which is all easy to use. Visit http://www.dovepress.com/testimonials.php to read real quotes from published authors.

Submit your manuscript here: http://www.dovepress.com/drug-design-development-and-therapy-journal 